\title{
Amenability and the Extension Property
}

\author{
Antoine Derighetti \\ EPFL SB-DO, MA A1 354, Station 8, CH-1015 Lausanne, Switzerland \\ Email: antoine.derighetti@epfl.ch
}

Received 4 September 2014; revised 26 September 2014; accepted 6 October 2014

Copyright (C) 2014 by author and Scientific Research Publishing Inc.

This work is licensed under the Creative Commons Attribution International License (CC BY).

http://creativecommons.org/licenses/by/4.0/

(c) () Open Access

\section{Abstract \\ Let $G$ be a locally compact group, $H$ a closed amenable subgroup and $u$ an element of the Herz Figà-Talamanca algebra of $H$ with compact support, we prove the existence of an extension of $u$ to $G$, with a good control of the norm and of the support of the extension.}

\section{Keywords}

\section{Convolution Operators, Locally Compact Groups, Abstract Harmonic Analysis, Amenable Groups}

\section{Introduction}

Let $G$ be a locally compact group and $H$ a closed subgroup, this paper is concerned with the problem of extending coefficients of the regular representation of $H$ to $G$. Suppose $H$ normal in G. In 1973 [1] C. Herz proved that for $u \in A_{p}(H)$ with compact support, for every $\varepsilon>0$ and for every $U$ neighborhood of suppu in $G$ there is $v \in A_{p}(G)$ with $\operatorname{Res}_{H} v=u,\|v\|<\|u\|+\varepsilon$ and supp $v \subset U$. In this work we want to treat the case of non normal subgroups. We succeed assuming that the subgroup $H$ is amenable (Theorem 5). C. Fiorillo obtained [2] already this result assuming however the unimodularity of $G$ and of $H$. But the $A N$ part of the Iwasawa decomposition of $S L_{2}(\mathbb{R})$ was out of reach. Even for $G$ amenable our result is new: the case of the non-normal copy of $\mathbb{R}$ in the $a x+b$-group was also out of reach.

Without control of norm and support of the extension, the theorem has been obtained in 1972 by McMullen [3]. With control of the norm, but not considering the supports, the statement is due Herz [1] (see also [4]).

\section{A Property of Amenable Subgroups}

We denote by $C_{00}(G)$ the set of all complex valued continuous functions on $G$ with compact support. We choose a positive continuous function $q$ on $G$ such that $q(x h)=q(x) \Delta_{H}(h) \Delta_{G}\left(h^{-1}\right)$, left invariant measures on $G$ and $H$ and a measure $d_{q} \dot{x}$ on $G / H$ as in Chapter 8 of [5]. The following Lemma will be used in the proof of our main result. See below the steps 1$)_{3}$ and 1$)_{4}$ of the proof of Lemma 2. 
Lemma 1 Let $G$ be a locally compact group, $H$ a closed amenable subgroup, $K$ a compact subset of $G, U$ a neighborhood of $e$ in $G$ and $\varepsilon>0$. Then there is $f \in C_{00}^{+}(G)$ such that $N_{1}(f)=1$, $\operatorname{supp} f \subset U$ and

$$
\int_{K}\left|\int_{H} f(h x) \Delta_{G}\left(h^{-1}\right) \mathrm{d} h-\int_{H} f(x h) \mathrm{d} h\right| \mathrm{d} x<\varepsilon
$$

Proof. Let $U_{0}$ be a compact neighborhood of $e$ in $G$ with $U_{0} \subset U, K_{1}=\left(U_{0} K^{-1} \cup K^{-1} U_{0}\right) \cap H$ and $\delta=\max _{h \in K_{1}} \Delta_{G}\left(h^{-1}\right)$ By the Proposition 2.1 of [6] (p. 463), there is $f \in C_{00}^{+}(G)$ such that $N_{1}(f)=1$, supp $f \subset U_{0}$ and such that $N_{1}\left(f_{h} \Delta_{G}(h)-_{h} f\right)<\varepsilon / 2 \delta m_{H}\left(K_{1}\right)$ for every $h \in K_{1}$. For every $x \in K$ we have

$$
\left|\int_{H} f(h x) \Delta_{G}\left(h^{-1}\right) \mathrm{d} h-\int_{H} f(x h) \mathrm{d} h\right| \leq \int_{H} 1_{K_{H}}(h)\left|f(h x) \Delta_{G}\left(h^{-1}\right)-f(x h)\right| \mathrm{d} h
$$

where $K_{H}=\left(\operatorname{supp} f K^{-1} \cup K^{-1} \operatorname{supp} f\right) \cap H$ Consequently

$$
\begin{aligned}
\int_{K}\left|\int_{H} f(h x) \Delta_{G}\left(h^{-1}\right) \mathrm{d} h-\int_{H} f(x h) \mathrm{d} h\right| \mathrm{d} x & \leq \int_{G} 1_{K}(x)\left(\int_{H} 1_{K_{H}}(h)\left|f(h x) \Delta_{G}\left(h^{-1}\right)-f(x h)\right| \mathrm{d} h\right) \mathrm{d} x \\
& \leq \int_{H} 1_{K_{H}}(h)\left(\int_{G}\left|f(h x) \Delta_{G}\left(h^{-1}\right)-f(x h)\right| \mathrm{d} x\right) \mathrm{d} h .
\end{aligned}
$$

\section{Approximation Theorem for Convolution Operators Supported by Subgroups}

We refer to [7] for $A_{p}(G), C V_{p}(G), P M_{p}(G)$ and the canonical map $i$ of $C V_{p}(H)$ into $C V_{p}(G)$ (Section 7.1 p. 101). We denote by $\mathcal{L}\left(L^{p}(G)\right)$ the Banach space of all bounded operators of $L^{p}(G)$.

We define a family of linear maps $\Lambda_{k, l}^{q}$ of $\mathcal{L}\left(L^{p}(G)\right)$ into $\mathcal{L}\left(L^{p}(H)\right)$ where $H$ is an arbitrary closed subgroup of $G$. We precise that $\tau_{p}$ is the involution of $L^{p}(G) \tau_{p}(f)(x)=f\left(x^{-1}\right) \Delta_{G}\left(x^{-1}\right)^{1 / p}$ and that for $k \in C_{00}(G), \varphi \in C_{00}(H)$ and $x \in G$ we have $\left(k *_{H} \varphi\right)(x)=\int_{H} k(x h) \varphi\left(h^{-1}\right) \mathrm{d} h$.

Definition 1. Let $G$ be a locally compact group, $H$ an arbitrary closed subgroup, $1<p<\infty$ and $k, l \in C_{00}(G)$. For $T \in \mathcal{L}\left(L^{p}(G)\right)$ we set for $\varphi, \psi \in C_{00}(H)$

$$
\left\langle\Lambda_{k, l}^{q}(T)[\varphi],[\psi]\right\rangle=\left\langle T\left[\tau_{p}\left(q^{1 / p}\left(k *_{H} \tau_{p} \varphi\right)\right)\right],\left[\tau_{p^{\prime}}\left(q^{1 / p^{\prime}}\left(k *_{H} \tau_{p^{\prime}} \psi\right)\right)\right]\right\rangle .
$$

Then $\Lambda_{k, l}^{q}(T) \in \mathcal{L}\left(L^{p}(H)\right)$ and $\left\|\Lambda_{k, l}^{q}(T)\right\| \leq\|T\| N_{p}\left(T_{H}|k|\right) N_{p^{\prime}}\left(T_{H}|l|\right) \quad$ where $\quad T_{H} k(\dot{x})=\int_{H} k(x h) \mathrm{d} h$. If $T \in C V_{p}(G)$ then $\Lambda_{k, l}^{q}(T) \in C V_{p}(H)$ and $\operatorname{supp} \Lambda_{k, l}^{q}(T)$ is contained in (suppk) $)^{-1} \operatorname{supp} T(\operatorname{suppl})$ [8].

Lemma 2. Let $G$ be a locally compact group, $H$ a closed amenable subgroup, $p>1$, $\varphi_{1}, \cdots, \varphi_{m}, \psi_{1}, \cdots, \psi_{m} \in C_{00}(H), \varepsilon>0$ and $U$ an open neighborhood of $e$ in $G$. Then there is $k, l \in C_{00}^{+}(G)$ with suppk $\subset U$, suppl $\subset U, N_{p}\left(T_{H} k\right) N_{p^{\prime}}\left(T_{H} l\right)<1+\varepsilon$ and such that

$$
\left|\left\langle\Lambda_{k, l}^{q}(i(S))\left[\varphi_{j}\right],\left[\psi_{j}\right]\right\rangle-\left\langle S\left[\varphi_{j}\right],\left[\psi_{j}\right]\right\rangle\right| \leq \varepsilon\|S\|
$$

for every $1 \leq j \leq m$ and every $S \in C V_{p}(H)$.

Proof. Let $0<\eta<1$ with $\eta<2^{-1} \varepsilon\left(4+N_{p}\left(\varphi_{j}\right)+N_{p^{\prime}}\left(\psi_{j}\right)\right)^{-1}$ for every $1 \leq j \leq m$. There is $U_{0}$ a compact symmetric neighborhood of $e$ in $G$ with $U_{0} \subset U$ and such that $\Delta_{H}(h)>(1+\eta)^{-1}$ for every $h \in U_{0}^{2} \cap H$. There is $V$ open neighborhood of $e$ in $H$ such that $N_{p}\left(\varphi_{j}-\left(\varphi_{j}\right)_{h^{-1}} \Delta_{H}\left(h^{-1}\right)\right)$ and $N_{p^{\prime}}\left(\psi_{j}-\left(\psi_{j}\right)_{h^{-1}} \Delta_{H}\left(h^{-1}\right)\right)$ are both smaller than $\eta / 2$ for every $1 \leq j \leq m$ and for every $h \in V$. We can choose $k^{\prime} \in C_{00}^{+}(G)$ with supp $k^{\prime} \subset U_{0}, \int_{H} k^{\prime}(h) \mathrm{d} h=1 \int_{H} k^{\prime}(x h) \mathrm{d} h \leq 1 \quad \int_{H} k^{\prime}(h x) \Delta_{H}\left(h^{-1}\right) \mathrm{d} h \leq 1$ for every $x \in G$ and such that suppk' $\cap H \subset V$.

Let $U_{1}$ be a symmetric compact neighborhood of $e$ in $G$ contained in $U_{0}$ with

$$
(1+\eta)^{-1}<\Delta_{G}(x)<1+\eta
$$


for every $x \in U_{1}$ and such that

$$
\begin{aligned}
& N_{p}\left(\operatorname{Res}_{H}\left(\varphi_{j} *_{H} k^{\prime}\right)-\left(\varphi_{j} *_{H} k^{\prime}\right)_{x, H}\right)<\eta / 2, \\
& N_{p^{\prime}}\left(\operatorname{Res}_{H}\left(\psi_{j} *_{H} k^{\prime}\right)-\left(\psi_{j} *_{H} k^{\prime}\right)_{x, H}\right)<\eta / 2
\end{aligned}
$$

for every $1 \leq j \leq m$ and for every $x \in U_{1}$ (for $f: G \rightarrow \mathbb{C}$ and $x \in G$ we denote by $f_{x, H}$ the function defined on $H$ by $h \mapsto f(x h))$.

We put $K=\bigcup_{j=1}^{m} \operatorname{supp} \varphi_{j} \cup \operatorname{supp} \psi_{j}, \quad A=\max _{x \in U_{1}}\left(T_{H} q^{-1 / p} \tau_{p} k^{\prime}\right)(\omega(x))$ and $B=\max _{x \in U_{1}}\left(T_{H} q^{-1 / p^{\prime}} \tau_{p^{\prime}} k^{\prime}\right)(\omega(x))$ where $\omega$ is the canonical map of $G$ onto $G / H$.

By the preceding Lemma there is $f \in C_{00}^{+}(G)$ with $\operatorname{supp} f \subset U_{1} \quad N_{1}(f)=1$ and such that

$$
\int_{\left(K U_{0}\right)^{-1}}\left|\int_{H} f(h x) \Delta_{G}\left(h^{-1}\right) \mathrm{d} h-\int_{H} f(x h) \mathrm{d} h\right| \mathrm{d} x
$$

is smaller than

$$
\frac{\eta^{p}}{2^{p}\left(1+\left\|\varphi_{j} *_{H} k^{\prime}\right\|_{\infty}\right)^{p} \max _{x \in K U_{0}} \Delta_{G}(x)\left(1+N_{p^{\prime}}\left(\psi_{j}\right) \max _{x \in U_{1}} q(x)^{1 / p^{\prime}} B\right)^{p}}
$$

and also smaller than

$$
\frac{\eta^{p^{\prime}}}{2^{p^{\prime}}\left(1+\left\|\psi_{j} *_{H} k^{\prime}\right\|_{\infty}\right)^{p^{\prime}}\left(1+N_{p}\left(\varphi_{j}\right)\right)^{p^{\prime}} \max _{x \in K U_{0}} \Delta_{G}(x)^{p^{\prime} / p} \max _{x \in U_{1}} q(x)^{p^{\prime} / p} A^{p^{\prime}}}
$$

for every $1 \leq j \leq m$. We finally put $F=\left(T_{H} f\right) \circ \omega \quad L=\left(T_{H} q^{-1} \breve{f}\right) \circ \omega, \quad k^{\prime \prime}=q^{-1 / p} F^{1 / p} \tau_{p} k^{\prime} \quad$ and $l^{\prime \prime}=q^{-1 / p^{\prime}} F^{1 / p^{\prime}} \tau_{p^{\prime}} k^{\prime}$.

1) For every $S \in C V_{p}(H)$ and every $1 \leq j \leq m$ we have

$$
\left|\left\langle\Lambda_{k^{\prime \prime}, l^{\prime}}^{q}(i(S))\left[\varphi_{j}\right],\left[\psi_{j}\right]\right\rangle-\left\langle i(S)\left[L^{1 / p} q^{1 / p}\left(\varphi_{j} *_{H} k^{\prime}\right)\right],\left[L^{1 / p^{\prime}} q^{1 / p^{\prime}}\left(\psi_{j} *_{H} k^{\prime}\right)\right]\right\rangle\right| \leq \eta\|S\| .
$$

1) We show at first that

$$
\begin{aligned}
& \left|\left\langle\Lambda_{k^{\prime \prime}, l^{\prime}}^{q}(i(S))\left[\varphi_{j}\right],\left[\psi_{j}\right]\right\rangle-\left\langle i(S)\left[L^{1 / p} q^{1 / p}\left(\varphi_{j} *_{H} k^{\prime}\right)\right],\left[L^{1 / p^{\prime}} q^{1 / p^{\prime}}\left(\psi_{j} *_{H} k^{\prime}\right)\right]\right\rangle\right| \\
& \leq \| \\
& \quad \leq S \| N_{p}\left(\left(\varphi_{j} *_{H} k^{\prime}\right)\left(\breve{F}^{1 / p}-L^{1 / p} q^{1 / p}\right)\right) N_{p^{\prime}}\left(\breve{F}^{1 / p^{\prime}}\left(\psi_{j} *_{H} k^{\prime}\right)\right) \\
& \quad+\|S\| N_{p}\left(q^{1 / p} \breve{L}^{1 / p^{\prime}}\left(\varphi_{j} *_{H} k^{\prime}\right)\right) N_{p^{\prime}}\left(\left(\psi_{j} *_{H} k^{\prime}\right)\left(\breve{F}^{1 / p^{\prime}}-L^{1 / p^{\prime}} q^{1 / p^{\prime}}\right)\right) .
\end{aligned}
$$

From

$$
\tau_{p}\left(q^{1 / p}\left(k^{\prime \prime} *_{H} \tau_{p} \varphi_{j}\right)\right)=\varphi_{j} *_{H}\left(\breve{F}^{1 / p} k^{\prime}\right)=\breve{F}^{1 / p}\left(\varphi_{j} *_{H} k^{\prime}\right)
$$

we obtain indeed

$$
\left\langle\Lambda_{k^{\prime \prime}, l^{\prime \prime}}^{q}(i(S))\left[\varphi_{j}\right],\left[\psi_{j}\right]\right\rangle=\left\langle i(S)\left[\breve{F}^{1 / p}\left(\varphi_{j} *_{H} k^{\prime}\right)\right],\left[\breve{F}^{1 / p^{\prime}}\left(\psi_{j} *_{H} k^{\prime}\right)\right]\right\rangle .
$$

1) $)_{2}$ For every $1 \leq j \leq m$ we have

$$
N_{p^{\prime}}\left(\breve{F}^{1 / p^{\prime}}\left(\psi_{j} *_{H} k^{\prime}\right)\right) \leq N_{p^{\prime}}\left(\psi_{j}\right) \max _{x \in U_{1}} q(x)^{1 / p^{\prime}} B .
$$

We have

$$
N_{p^{\prime}}\left(\breve{F}^{1 / p^{\prime}}\left(\psi_{j} *_{H} k^{\prime}\right)\right)^{p}=\int_{G / H} T_{H} f(\dot{x})\left(\int_{H}\left|\tau_{p^{\prime}}\left(\psi_{j} *_{H} k^{\prime}\right)(x h)\right|^{p^{\prime}} q(x h)^{-1} \mathrm{~d} h\right) \mathrm{d}_{q} \dot{x} .
$$

But for every $x \in G$

$$
\frac{\left|\tau_{p^{\prime}}\left(\psi_{j} *_{H} k^{\prime}\right)(x h)\right|^{p^{\prime}}}{q(x h)}=\left|\left(\tau_{p^{\prime}}\left(\breve{q}^{-1 / p^{\prime}} k^{\prime}\right) *_{H} \tau_{p^{\prime}} \psi_{j}\right)(x h)\right|^{p^{\prime}}
$$


and therefore

$$
\int_{H} \frac{\left|\tau_{p^{\prime}}\left(\psi_{j} *_{H} k^{\prime}\right)(x h)\right|^{p^{\prime}}}{q(x h)} \mathrm{d} h=N_{p^{\prime}}\left(\left(\tau_{p^{\prime}}\left(\breve{q}^{-1 / p^{\prime}} k^{\prime}\right)\right)_{x, H} * \tau_{p^{\prime}} \psi_{j}\right)^{p^{\prime}}
$$

consequently

$$
\begin{aligned}
N_{p^{\prime}}\left(\breve{F}^{1 / p^{\prime}}\left(\psi_{j} *_{H} k^{\prime}\right)\right)^{p^{\prime}} & \leq N_{p^{\prime}}\left(\psi_{j}\right)^{p^{\prime}} \int_{G / H} T_{H} f(\dot{x}) N_{1}\left(\tau_{p^{\prime}}\left(\breve{q}^{-1 / p^{\prime}} k^{\prime}\right)_{x, H}\right)^{p^{\prime}} \mathrm{d}_{q} \dot{x} \\
& =N_{p^{\prime}}\left(\psi_{j}\right)^{p^{\prime}} \int_{G} q(x) f(x)\left(T_{H}\left(q^{-1 / p^{\prime}} \tau_{p^{\prime}} k^{\prime}\right)(\omega(x))\right)^{p^{\prime}} \mathrm{d} x .
\end{aligned}
$$

1) $)_{3}$ For every $1 \leq j \leq m$ we have

$$
\begin{aligned}
N_{p}\left(\left(\varphi_{j} *_{H} k^{\prime}\right)\left(\breve{F}^{1 / p}-L^{1 / p} q^{1 / p}\right)\right) \leq & \left\|\varphi_{j} *_{H} k^{\prime}\right\|_{\infty} \max _{x \in K U_{0}} \Delta_{G}(x)^{1 / p} \\
& \times\left(\int_{\left(K U_{0}\right)^{-1}}\left|\int_{H} f(h x) \Delta_{G}\left(h^{-1}\right) \mathrm{d} h-\int_{H} f(x h) \mathrm{d} h\right| \mathrm{d} x\right)^{1 / p} .
\end{aligned}
$$

As above

$$
N_{p}\left(\left(\varphi_{j} *_{H} k^{\prime}\right)\left(\breve{F}^{1 / p}-L^{1 / p} q^{1 / p}\right)\right)^{p} \leq \int_{G}\left|\left(\varphi_{j} *_{H} k^{\prime}\right)(x)\right|^{p}|\breve{F}(x)-q(x) L(x)| \mathrm{d} x
$$

taking in account that $q(x) L(x)=\int_{H} f\left(h x^{-1}\right) \Delta_{G}\left(h^{-1}\right) \mathrm{d} h$ we obtain

$$
N_{p}\left(\left(\varphi_{j} *_{H} k^{\prime}\right)\left(\breve{F}^{1 / p}-L^{1 / p} q^{1 / p}\right)\right)^{p} \leq \int_{G}\left|\left(\varphi_{j} *_{H} k^{\prime}\right)\left(x^{-1}\right)\right|^{p} \Delta_{G}\left(x^{-1}\right)\left|\int_{H} f(h x) \Delta_{G}\left(h^{-1}\right) \mathrm{d} h-\int_{H} f(x h) \mathrm{d} h\right| \mathrm{d} x .
$$

$1)_{4}$ Proof of 1). Using 1$)_{3}$ and 1$)_{2}$ one obtains an estimate for $N_{p}\left(q^{1 / p} L^{1 / p}\left(\varphi_{j} *_{H} k^{\prime}\right)\right)$. We finish then the proof of 1) using 1$)_{1}$.

2) For every $S \in C V_{p}(H)$ and every $1 \leq j \leq m$ we have

$$
\begin{aligned}
& \left|\left\langle i(S)\left[L^{1 / p} q^{1 / p}\left(\varphi_{j} *_{H} k^{\prime}\right)\right],\left[L^{1 / p^{\prime}} q^{1 / p^{\prime}}\left(\psi_{j} *_{H} k^{\prime}\right)\right]\right\rangle-\int_{G} \breve{f}(x) \mathrm{d} x\left\langle S\left[\varphi_{j}\right],\left[\psi_{j}\right]\right\rangle\right| \\
& \quad \leq \eta\|S\| \int_{G} \breve{f}(x) \mathrm{d} x\left(1+N_{p}\left(\varphi_{j}\right)+N_{p^{\prime}}\left(\psi_{j}\right)\right) .
\end{aligned}
$$

By the Corollary 6 of section 7.2 p.112 of [7]

$$
\begin{aligned}
& \left\langle i(S)\left[L^{1 / p} q^{1 / p}\left(\varphi_{j} *_{H} k^{\prime}\right)\right],\left[L^{1 / p^{\prime}} q^{1 / p^{\prime}}\left(\psi_{j} *_{H} k^{\prime}\right)\right]\right\rangle \\
& =\int_{G}\left(T_{H, q} \breve{f}\right)(\omega(x)) \beta(x) q(x)\left\langle S\left[\left(\varphi_{j} *_{H} k^{\prime}\right)_{x, H}\right],\left[\left(\psi_{j} *_{H} k^{\prime}\right)_{x, H}\right]\right\rangle \mathrm{d} x .
\end{aligned}
$$

Consequently

$$
\begin{aligned}
& \left|\left\langle i(S)\left[L^{1 / p} q^{1 / p}\left(\varphi_{j} *_{H} k^{\prime}\right)\right],\left[L^{1 / p^{\prime}} q^{1 / p^{\prime}}\left(\psi_{j} *_{H} k^{\prime}\right)\right]\right\rangle-\int_{G} \breve{f}(x) \mathrm{d} x\left\langle S\left[\varphi_{j}\right],\left[\psi_{j}\right]\right\rangle\right| \\
& \quad \leq \int_{G}\left(T_{H, q} \breve{f}\right)(\omega(x)) \beta(x) q(x) 1_{U_{1} H}(x)\left|\left\langle S\left[\left(\varphi_{j} *_{H} k^{\prime}\right)_{x, H}\right],\left[\left(\psi_{j} *_{H} k^{\prime}\right)_{x, H}\right]\right\rangle-\left\langle S\left[\varphi_{j}\right],\left[\psi_{j}\right]\right\rangle\right| \mathrm{d} x .
\end{aligned}
$$

But by definition of $U_{1}$ for every $x \in U_{1} H$ we have

$$
\left|\left\langle S\left[\left(\varphi_{j} *_{H} k^{\prime}\right)_{x, H}\right],\left[\left(\psi_{j} *_{H} k^{\prime}\right)_{x, H}\right]\right\rangle-\left\langle S\left[\varphi_{j}\right],\left[\psi_{j}\right]\right\rangle\right| \leq \eta\|S\|\left(1+N_{p}\left(\varphi_{j}\right)+N_{p^{\prime}}\left(\psi_{j}\right)\right) .
$$

3) End of the proof of Lemma 2. We are now able to define the functions $k$ and $l$ of the Lemma $k=\left(\int_{G} \breve{f}(x) \mathrm{d} x\right)^{-1 / p} k^{\prime \prime}$ and $l=\left(\int_{G} \breve{f}(x) \mathrm{d} x\right)^{-1 / p^{\prime}} l^{\prime \prime}$. Using 1) and 2) we get

$$
\begin{aligned}
\left|\left\langle\Lambda_{k, l}^{q}(i(S))\left[\varphi_{j}\right],\left[\psi_{j}\right]\right\rangle-\left\langle S\left[\varphi_{j}\right],\left[\psi_{j}\right]\right\rangle\right| & \leq\left(\int_{G} \breve{f}(x) \mathrm{d} x\right)^{-1} \eta\|S\|+\eta\|S\|\left(1+N_{p}\left(\varphi_{j}\right)+N_{p^{\prime}}\left(\psi_{j}\right)\right) \\
& \leq(1+\eta) \eta\|S\|+\left(1+N_{p}\left(\varphi_{j}\right)+N_{p^{\prime}}\left(\psi_{j}\right)\right)\|S\| \eta \\
& \leq \eta\|S\|\left(3+N_{p}\left(\varphi_{j}\right)+N_{p^{\prime}}\left(\psi_{j}\right)\right) \leq \varepsilon\|S\| .
\end{aligned}
$$


Clearly suppk $\subset U$ and $\operatorname{supp} l \subset U$. It remains to show that $N_{p}\left(T_{H} k\right) N_{p^{\prime}}\left(T_{H} l\right)<1+\varepsilon$. We have

$$
N_{p}\left(T_{H} k^{\prime \prime}\right)^{p}=\int_{G} f(x) 1_{U_{1}}(x) \Delta_{G}\left(x^{-1}\right)\left(\int_{H} k^{\prime}\left(h x^{-1}\right) \Delta_{H}\left(h^{-1}\right)^{1 / p^{\prime}} \mathrm{d} h\right)^{p} \mathrm{~d} x .
$$

But for $x \in U_{1}$

$$
\int_{H} k^{\prime}\left(h x^{-1}\right) \Delta_{H}\left(h^{-1}\right)^{1 / p^{\prime}} \mathrm{d} h \leq(1+\eta)^{1 / p}
$$

hence $N_{p}\left(T_{H} k\right)<(1+\eta)^{3 / p}$ and similarly $N_{p^{\prime}}\left(T_{H} l\right)<(1+\eta)^{3 / p^{\prime}}$, we finally get $N_{p}\left(T_{H} k\right) N_{p^{\prime}}\left(T_{H} l\right)<1+\varepsilon$.

Theorem 3 Let $G$ be a locally compact group, $H$ a closed amenable subgroup, $p>1,\left(r_{n}\right)$ a sequence of $\mathcal{L}^{p}(H),\left(s_{n}\right)$ a sequence of $\mathcal{L}^{p^{\prime}}(H), \varepsilon>0$ and $U$ an open neighborhood of $e$ in $G$. Suppose that the series $\sum N_{p}\left(r_{n}\right) N_{p^{\prime}}\left(s_{n}\right)$ converges. Then there is $k, l \in C_{00}^{+}(G)$ with suppk $\subset U$, suppl $\subset U$, $N_{p}\left(T_{H} k\right) N_{p^{\prime}}\left(T_{H} l\right)<1$ and such that

$$
\sum_{n=1}^{\infty}\left|\left\langle\Lambda_{k, l}^{q}(i(S))\left[r_{n}\right],\left[s_{n}\right]\right\rangle-\left\langle S\left[r_{n}\right],\left[s_{n}\right]\right\rangle\right| \leq \varepsilon\|S\|
$$

for every $S \in C V_{p}(H)$

Proof. We choose $0<\eta<1$ with $\eta<\varepsilon\left(1+\sum N_{p}\left(r_{n}\right) N_{p^{\prime}}\left(s_{n}\right)\right)^{-1}$.

1) There is $k^{\prime}, l^{\prime} \in C_{00}^{+}(G)$ with $\operatorname{supp}^{\prime} \subset U$ suppl $l^{\prime} \subset U, N_{p}\left(T_{H} k^{\prime}\right) N_{p^{\prime}}\left(T_{H} l^{\prime}\right)<1+\eta$ and such that

$$
\sum_{n=1}^{\infty}\left|\left\langle\Lambda_{k^{\prime}, l^{\prime}}^{q}(i(S))\left[r_{n}\right],\left[s_{n}\right]\right\rangle-\left\langle S\left[r_{n}\right],\left[s_{n}\right]\right\rangle\right| \leq \eta\|S\|
$$

for every $S \in C V_{p}(H)$.

There are $\left(\varphi_{n}\right)$ and $\left(\psi_{n}\right)$ sequences of $C_{00}(H)$ with

$$
N_{p}\left(r_{n}-\varphi_{n}\right)<\frac{\eta}{3^{2} 2^{n+1}\left(1+N_{p^{\prime}}\left(s_{n}\right)\right)}
$$

and

$$
N_{p^{\prime}}\left(s_{n}-\psi_{n}\right)<\frac{\eta}{3^{2} 2^{n+1}\left(1+N_{p}\left(r_{n}\right)\right)}
$$

for every $n \in \mathbb{N}$. From the convergence of $\sum N_{p}\left(\varphi_{n}\right) N_{p^{\prime}}\left(\psi_{n}\right)$ follows the existence of $N \in \mathbb{N}$ such that

$$
\sum_{n=N+1}^{\infty} N_{p}\left(\varphi_{n}\right) N_{p^{\prime}}\left(\psi_{n}\right)<\eta / 9 .
$$

By Lemma 2 there is $k^{\prime}, l^{\prime} \in C_{00}^{+}(G)$ with $\operatorname{supp} k^{\prime} \subset U, \quad \operatorname{supp} l^{\prime} \subset U$,

$$
N_{p}\left(T_{H} k^{\prime}\right) N_{p^{\prime}}\left(T_{H} l^{\prime}\right)<1+\frac{\eta}{3 N}
$$

and such that

$$
\left|\left\langle\Lambda_{k^{\prime}, l^{\prime}}^{q}(i(S))\left[\varphi_{j}\right],\left[\psi_{j}\right]\right\rangle-\left\langle S\left[\varphi_{j}\right],\left[\psi_{j}\right]\right\rangle\right| \leq \frac{\eta\|S\|}{3 N}
$$

for every $1 \leq n \leq N$ and every $S \in C V_{p}(H)$. Consequently

$$
\begin{aligned}
\sum_{n=1}^{\infty}\left|\left\langle\Lambda_{k^{\prime}, l^{\prime}}^{q}(i(S))\left[r_{n}\right],\left[s_{n}\right]\right\rangle-\left\langle S\left[r_{n}\right],\left[s_{n}\right]\right\rangle\right| & \leq \frac{\eta\|S\|}{3}+\sum_{n=1}^{\infty}\left|\left\langle\Lambda_{k^{\prime}, l^{\prime}}^{q}(i(S))\left[\varphi_{j}\right],\left[\psi_{j}\right]\right\rangle-\left\langle S\left[\varphi_{j}\right],\left[\psi_{j}\right]\right\rangle\right| \\
& \leq \frac{\eta\|S\|}{3}+\frac{2 \eta\|S\|}{3} .
\end{aligned}
$$

2) End of the proof of Theorem 3. It suffices to put $k=k^{\prime}(1+\eta)^{-1 / p}$ and $l=l^{\prime}(1+\eta)^{-1 / p^{\prime}}$ to obtain 


$$
\begin{aligned}
& N_{p}\left(T_{H} k\right) N_{p^{\prime}}\left(T_{H} l\right)<1 \text { and } \\
& \qquad \sum_{n=1}^{\infty}\left|\left\langle\Lambda_{k, l}^{q}(i(S))\left[r_{n}\right],\left[s_{n}\right]\right\rangle-\left\langle S\left[r_{n}\right],\left[s_{n}\right]\right\rangle\right| \leq \frac{\eta}{1+\eta}\|S\|\left(1+\sum_{n=1}^{\infty} N_{p}\left(r_{n}\right) N_{p^{\prime}}\left(s_{n}\right)\right) \leq \varepsilon\|S\| .
\end{aligned}
$$

\section{The Main Result}

Definition 2 Let $G$ be a locally compact group, $H$ an arbitrary closed subgroup, $1<p<\infty$ and $k, l \in C_{00}(G)$ For $u \in A_{p}(H)$ we put

$$
\Phi_{k, l}^{q}(u)=\sum_{n=1}^{\infty} \overline{\left(q^{1 / p}\left(k *_{H} \varphi_{n}\right)\right)} *_{G}\left(q^{1 / p^{\prime}}\left(l *_{H} \psi_{n}\right)\right)^{\vee}
$$

where $\left(\varphi_{n}\right)$ and $\left(\psi_{n}\right)$ are sequences of $C_{00}(H)$ such that $\sum N_{p}\left(\varphi_{n}\right) N_{p^{\prime}}\left(\psi_{n}\right)$ converges and such that $u=\sum \bar{\varphi}_{n} * \breve{\psi}_{n}$.

Then $\Phi_{k, l}^{q}$ is a linear map of $A_{p}(H)$ into $A_{p}(G)$, for $u \in A_{p}(H)$ and $T \in P M_{p}(G)$ one has $\left\langle u, \Lambda_{k, l}^{q}(T)\right\rangle=\left\langle\Phi_{k, l}^{q}(u), T\right\rangle, \Lambda_{k, l}^{q}(T) \in P M_{p}(H)$ and $\operatorname{supp} \Phi_{k, l}^{q}(u) \subset \operatorname{supp} k \operatorname{supp} u(\operatorname{suppl})^{-1} \quad$ [8].

Corollary 4 Let $G$ be a locally compact group, $H$ a closed amenable subgroup, $p>1$, $u \in A_{p}(H) \cap C_{00}(H), \varepsilon>0$ and $\Omega$ a neighborhood of suppu in $G$. Then there are $k, l \in C_{00}^{+}(G)$ with $\left\|\Phi_{k, l}^{q}(u)\right\| \leq\|u\|, \quad \operatorname{supp} \Phi_{k, l}^{q}(u) \subset \Omega$ and $\left\|\operatorname{Res}_{H} \Phi_{k, l}^{q}(u)-u\right\|<\varepsilon$.

Proof. There are sequences $\left(r_{n}\right),\left(s_{n}\right)$ of $C_{00}(H)$ such that $\sum N_{p}\left(r_{n}\right) N_{p^{\prime}}\left(s_{n}\right)$ converges and such that $u=\sum \bar{r}_{n} * \breve{s}_{n}$. Let $U$ be an open neighborhood of $e$ in $G$ such that $U$ suppu $U^{-1} \subset \Omega$. By Theorem 3 there is $k, l \in C_{00}^{+}(G)$ with suppk $\subset U$, suppl $\subset \in U, N_{p}\left(T_{H} k\right) N_{p^{\prime}}\left(T_{H} l\right)<1$ and such that

$$
\sum_{n=1}^{\infty}\left|\left\langle\Lambda_{k, l}^{q}(i(S))\left[\tau_{p} r_{n}\right],\left[\tau_{p^{\prime}} s_{n}\right]\right\rangle-\left\langle S\left[\tau_{p} r\right]_{n},\left[\tau_{p^{\prime}} s_{n}\right]\right\rangle\right| \leq \frac{\varepsilon}{2}\|S\|
$$

for every $S \in C V_{p}(H)$.

Consider an arbitrary $S \in P M_{p}(H)$ with $\|S\| \leq 1$. From

$$
\langle u, S\rangle=\sum_{n=1}^{\infty} \overline{\left\langle S\left[\tau_{p} r_{n}\right],\left[\tau_{p^{\prime}} S_{n}\right]\right\rangle}
$$

and

$$
\left\langle u, \Lambda_{k, l}^{q}(i(S))\right\rangle=\sum_{n=1}^{\infty} \overline{\left\langle\Lambda_{k, l}^{q}(i(S))\left[\tau_{p} r_{n}\right],\left[\tau_{p^{\prime}} s_{n}\right]\right\rangle}
$$

we get $\left|\left\langle u-\operatorname{Res}_{H} \Phi_{k, l}(u), S\right\rangle\right| \leq \varepsilon / 2$ and therefore $\left\|u-\operatorname{Res}_{H} \Phi_{k, l}(u)\right\|<\varepsilon$.

The following theorem is the main result of the paper.

Theorem 5 Let $G$ be a locally compact group, $H$ a closed amenable subgroup, $p>1$,

$u \in A_{p}(H) \cap C_{00}(H), \varepsilon>0$ and $\Omega$ a neighborhood of suppu in $G$. Then there is $v \in A_{p}(G) \cap C_{00}(G)$ with $\operatorname{Res}_{H} v=u\|v\|<\|u\|+\varepsilon$ and $\operatorname{supp} v \subset \Omega$

Proof. This proof is identical with the one of Proposition 1 ii) p. 115 of [1]. Let $\Omega^{\prime}$ be an open neighborhood of suppu in $G$ such that the closure of $\Omega^{\prime}$ in $G$ is compact and contained in $\Omega$. Using the Corollary 4 we show by induction the existence of a sequence $\left(u_{n}\right)$ of $A_{p}(H) \cap C_{00}(H)$ and of a sequence $\left(v_{n}\right)$ of $A_{p}(G) \cap C_{00}(G)$ such that $u_{1}=u, \quad \operatorname{supp} u_{n} \subset \Omega^{\prime}, \quad \operatorname{supp} v_{n} \subset \Omega^{\prime},\left\|v_{n}\right\| \leq\left\|u_{n}\right\|$, $\left\|u_{n}-\operatorname{Res}_{H} v_{n}\right\|<2^{-(n+1)} \varepsilon$ and $u_{n+1}=u_{n}-\operatorname{Res}_{H} v_{n}$ The function $\sum v_{n}$ satisfies all the requirements.

\section{References}

[1] Herz, C.S. (1973) Harmonic Synthesis for Subgroups. Annales de l'institut Fourier, 23, 91-123. http://dx.doi.org/10.5802/aif.473

[2] Fiorillo, C. (2009) An Extension Property for the Figà-Talamanca Herz Algebra. Proceedings of the American Mathematical Society, 137, 1001-1011. http://dx.doi.org/10.1090/S0002-9939-08-09679-2 
[3] McMullen, J.R. (1972) Extensions of Positive-Definite Functions. Memoirs of the American Mathematical Society, 117.

[4] Delaporte, J. and Derighetti, A. (1992) On Herz’ Extension Theorem. Bollettino dell'Unime Matematica Italiana, (7) 6-A, 245-247.

[5] Reiter, H. and Stegman, J.D. (2000) Classical Harmonic Analysis and Locally Compact Groups. Clarendon Press, Oxford.

[6] Derighetti, A. (2004) On Herz's Projection Theorem. Illinois Journal of Mathematics, 48, 463-476.

[7] Derighetti, A. (2011) Convolution Operators on Groups. Lecture Notes of the Unione Matematica Italiana, 11, Springer-Verlag, Berlin, Heidelberg.

[8] Delaporte, J. and Derighetti, A. (1995) p-Pseudomeasures and Closed Subgroups. Monatshefte für Mathematik, 119, 37-47. http://dx.doi.org/10.1007/BF01292767 
Scientific Research Publishing (SCIRP) is one of the largest Open Access journal publishers. It is currently publishing more than 200 open access, online, peer-reviewed journals covering a wide range of academic disciplines. SCIRP serves the worldwide academic communities and contributes to the progress and application of science with its publication.

Other selected journals from SCIRP are listed as below. Submit your manuscript to us via either submit@scirp.org or Online Submission Portal.
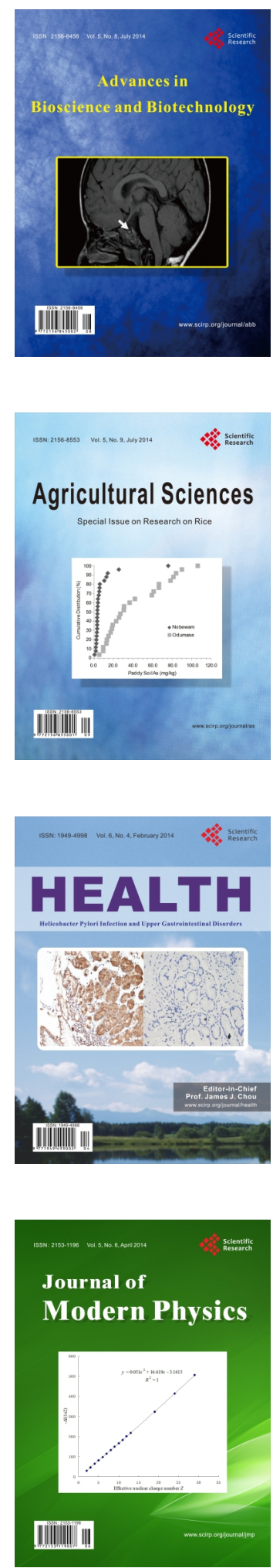
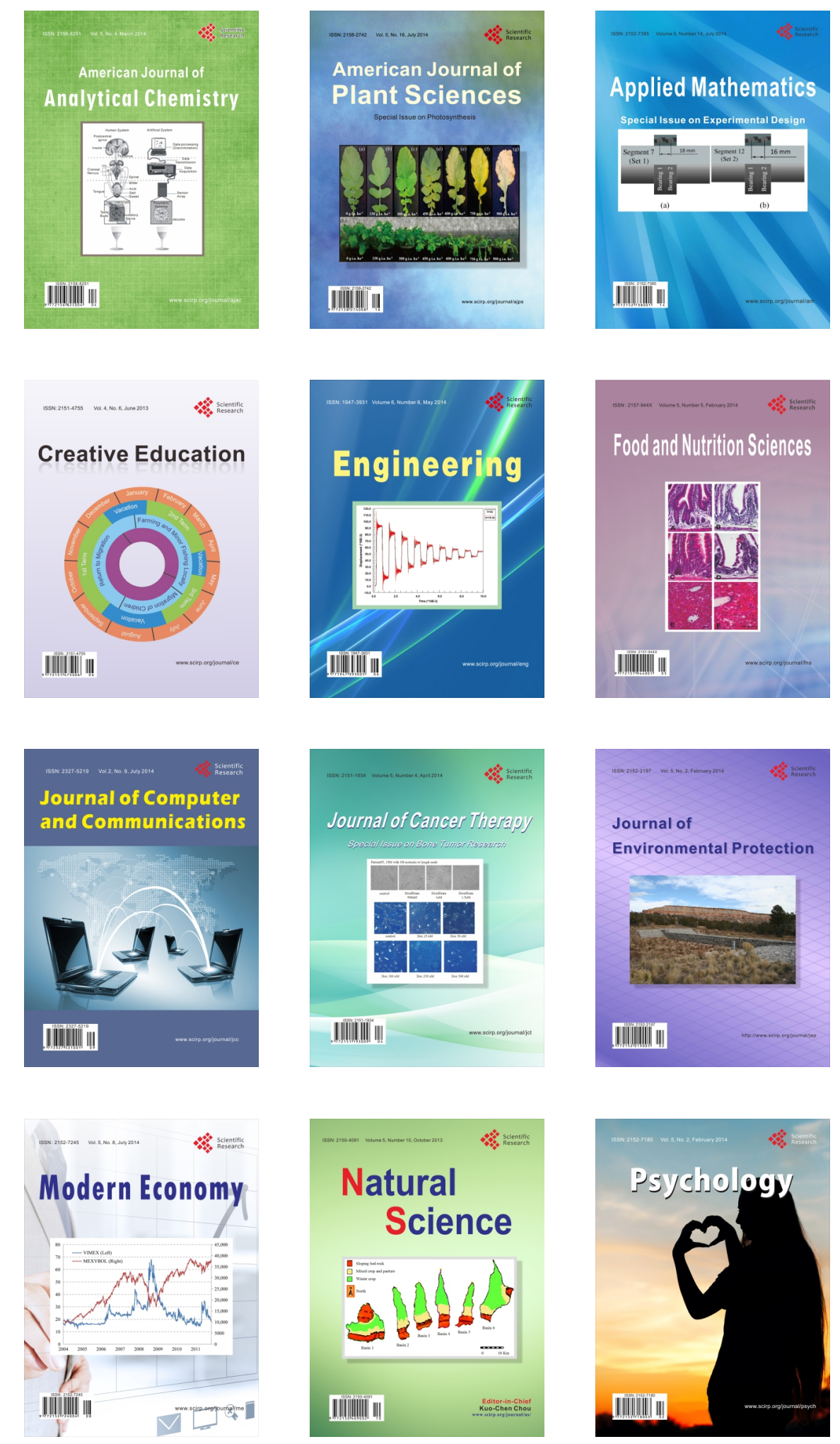